\title{
The quantum theory of Chern-Simons supergravity
}

\section{Andrianopoli, ${ }^{a, c, d}$ B.L. Cerchiai, ${ }^{a, c, e}$ P.A. Grassi ${ }^{b, c, d}$ and M. Trigiante ${ }^{a, c, d}$}

${ }^{a}$ DISAT, Politecnico di Torino, C.so Duca degli Abruzzi, 24, 10129 Torino, Italy

${ }^{b}$ Dipartimento di Scienze e Innovazione Tecnologica, Università del Piemonte Orientale, viale T. Michel, 11, 15121 Alessandria, Italy

${ }^{c}$ INFN, Sezione di Torino, via P. Giuria 1, 10125 Torino, Italy

${ }^{d}$ Arnold-Regge Center, via P. Giuria 1, 10125 Torino, Italy

${ }^{e}$ Centro Fermi, Museo Storico della Fisica e Centro Studi e Ricerche "Enrico Fermi", Piazza del Viminale 1, 00184 Roma, Italy

E-mail: laura.andrianopoli@polito.it, bianca.cerchiai@polito.it, pietro.grassi@uniupo.it, mario.trigiante@polito.it

Abstract: We consider $A d S_{3} N$-extended Chern-Simons supergravity (à la AchucarroTownsend) and we study its gauge symmetries. We promote those gauge symmetries to a BRST symmetry and we perform its quantization by choosing suitable gauge-fixings. The resulting quantum theories have different features which we discuss in the present work. In particular, we show that a special choice of the gauge-fixing correctly reproduces the Ansatz by Alvarez, Valenzuela and Zanelli for the graphene fermion.

KeYwords: BRST Quantization, Chern-Simons Theories

ArXiv EPrint: 1903.04431 


\section{Contents}

1 Introduction 1

$2 \quad \mathrm{D}=3 \mathrm{~N}$-extended Chern-Simons supergravity 5

2.1 Basic facts 5

$\begin{array}{ll}2.2 \text { A model for graphene from } \mathrm{D}=3 \text { supergravity } & 7\end{array}$

2.3 BRST formulation of $N=2 A d S_{3}$ supergravity 8

$\begin{array}{lll}2.4 \text { A secondary BRST symmetry } & 11\end{array}$

$\begin{array}{lll}2.5 & \text { Vector BRST symmetry } & 12\end{array}$

3 Gauge-fixing choices $\quad 13$

3.1 Counting of D.O.F.'s 13

$\begin{array}{ll}3.2 \text { Landau gauge-fixing and } \mathcal{N}=4 \text { supersymmetry } & 15\end{array}$

$\begin{array}{lll}3.3 & \text { Feynman gauge fixing and mass deformations } & 16\end{array}$

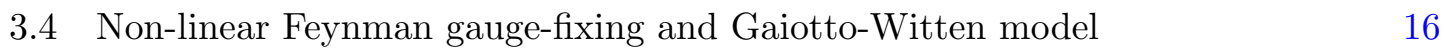

$\begin{array}{ll}3.5 & \text { Unconventional gauge fixing and AVZ Ansatz } \\ \end{array}$

$\begin{array}{ll}3.6 \bar{s} \text {-gauge fixing } & 17\end{array}$

4 Conclusions and outlook $\quad 18$

\section{Introduction}

It is tantalising to relate models which are apparently very different. Some years ago, Gaiotto and Witten, in [1], considered a Chern-Simons (CS) gauge theory in three dimensions coupled to $\mathcal{N}=2$ supersymmetric multiplets whose scalar components are coordinates of a hyper-Kähler manifold. The model descends from a four dimensional one in the presence of a defect and the potential is chosen to enhance the supersymmetry from $\mathcal{N}=2$ to $\mathcal{N}=4$. The conditions under which this is possible are certain relations between the moment maps associated with the linear action of the gauge group on the hyper-Kähler manifold which unveil a superalgebra hidden in the model.

They argued that the supersymmetric Wilson loops can be constructed in terms of that super algebra, but they did not elaborate further along these lines. In another paper [2], Kapustin and Saulina showed that Rozansky-Witten theory [3] coupled to a Chern-Simons gauge field can be written (up to an exact BRST term) as a Chern-Simons gauge theory on a supergroup. In particular they showed the following relation

Chern-Simons theory $\left.\right|_{\mathcal{S G}}+s$ (gauge fixing of fermion gauge symmetries) $=$

topologically twisted super-Chern-Simons theory $\left.\right|_{\mathcal{G}}+$ SUSY matter fields . 
where Chern-Simons theory $\left.\right|_{\mathcal{S G}}$ is a Chern-Simons theory on the supergroup $\mathcal{S G}$ (see also [4] for a complete discussion) plus a gauge-fixing and it can be recast in the form of a $\mathcal{N}=4$ super-Chern-Simons theory on the group $\mathcal{G}$ (which is the bosonic subgroup of $\mathcal{S G}$ ) coupled to $\mathcal{N}=4$ hyper-multiplets. The gauginos and the scalar fields of the $\mathcal{N}=4$ Chern-Simons multiplet are not dynamical and their equations of motion determine them in terms of the scalars and fermions in the hyper-multiplet sector. By inserting this solution back in the action, one gets additional non-trivial terms for the potential.

The complete scalar potential in the lower part of the correspondence (1.1) is incorporated into the gauge-fixing of the fermionic symmetry inside $\mathcal{S G}$ in the upper part of the same relation. The mapping from the lower to the upper part has been used in [5-8], to compute supersymmetric Wilson loops in terms of the Chern-Simons theory on the supergroup instead of the supersymmetric Chern-Simons theory.

In the correspondence (1.1) the $\mathcal{N}=4$ supersymmetry in the supersymmetric ChernSimons model originates, via a topological twist, from the BRST invariance of the upper theory in which only the fermionic gauge symmetries are covariantly fixed. It is important to emphasize that, in Chern-Simons theories, this invariance comprises, apart from the ordinary BRST and anti-BRST transformations $s, \bar{s}$, also "vector" symmetry transformations $s_{\mu}, \bar{s}_{\mu}$, as found in [15-18]. The twisted $\mathcal{N}=4$ world-volume supersymmetry in the lower-side of the relation (1.1) can be understood in terms of the whole set of these invariances, as we shall discuss in the present paper.

One of the purposes of this work is to apply this construction to a specific ChernSimons theory which describes $\mathcal{N}=2$ supersymmetric $\mathrm{AdS}_{3}$ supergravity, as shown by Achucarro and Townsend in [9].

Indeed, some years ago, Achucarro and Townsend observed that, in three dimensions, $N$-extended supergravity in the presence of a negative cosmological constant can be rewritten as a Chern-Simons theory whose gauge fields take values in the superalgebra $\mathfrak{o} \mathfrak{s p}(p \mid 2) \times \mathfrak{o s p}(q \mid 2)$ where $p+q=N$. The bosonic subalgebra is $\mathfrak{s o}(p) \otimes \mathfrak{s o}(q) \otimes \mathfrak{s p}(2) \otimes \mathfrak{s p}(2)$ and the gauge fields associated with the two $\mathfrak{s p}(2)$ 's are given in terms of the vielbein and the spin connection of the three dimensional manifold. The "gravitinos" (the gauge fields associated with the fermionic gauge generators) are in the bifundamental representations of $\mathrm{SO}(p) \times \mathrm{Sp}(2)$ and/or $\mathrm{SO}(q) \times \mathrm{Sp}(2)$ subgroups. The supersymmetry is realized as a gauge symmetry and therefore the fermionic charges are related to the fermionic (anticommuting) gauge fields. Being a Chern-Simons theory it does not depend on the 3 -dimensional metric on the world-volume. The latter however emerges from the gauge fixing, within BRST-exact terms in the Lagrangian. AdS-supergravity in three dimensions, being a Chern-Simons gauge theory on a supergroup, is a viable context where to apply the relation proposed by $[1,2]$. The structure of Achucarro and Townsend supergravity from a mathematical point of view is reminiscent of the ABJM model [10], which is a difference of two CS actions as well.

In $[1,2]$, the duality is based on a topological twist, where the twist is realized by taking the diagonal subgroup of the product of the original Lorentz group with an $\operatorname{Sp}(2)$ part of the R-symmetry. On a AdS background, instead, the analogous topological twist is naturally related to the (non-unique) choice of the Lorentz subalgebra inside the anti-de 
Sitter isometry $\mathfrak{s o}(2,2) \simeq \mathfrak{s p}(2) \times \mathfrak{s p}(2)$. Indeed, in this framework this topological twist corresponds to trading the antisymmetric part of the torsion $\tau$ for a cosmological constant $\Lambda$. More precisely, the choice as Lorentz group of one of the two $\operatorname{Sp}(2)$ factors - which corresponds to the untwisted theory in the flat background - is here associated with a non-vanishing space-time torsion $\tau \neq 0$. On the other hand, choosing instead the diagonal subgroup $\mathrm{SO}(1,2)_{D} \subset \mathrm{SO}(2,2)$ as Lorentz group - which corresponds to the topologically twisted theory in a flat background - is here associated with the choice of a torsionless spin-connection in a background with cosmological constant $\Lambda=-\tau^{2} \neq 0$.

The first step towards constructing the super-Chern-Simons theory coupled to matter fields for Achucarro-Townsend supergravity is to perform a suitable gauge-fixing of the fermionic part of the super-gauge symmetry. The gauge symmetry at the quantum level is replaced by the BRST symmetry and the gauge parameters are replaced by the ghost fields which, in the present case in which the gauge fixed symmetries are of fermionic type, are commuting scalar fields. To complete the gauge-fixing procedure, one needs an auxiliary sector, also known as an anti-ghost sector, which, in this case, consists of a set of commuting scalar fields and a set of fermionic Nakanishi-Lautrup fields [2]. The ghosts and the antighosts are commuting scalar fields belonging to conjugated representations of the bosonic gauge group $\mathcal{G}$. In fact they turn out to span a quaternionic-Kähler manifold which carries a tri-holomorphic action of $\mathcal{G}$. Finally, using the work of Kapustin and Saulina [2], we translate the degrees of freedom of the Chern-Simons supergravity in terms of the ones of a super-Chern-Simons theory with $\mathcal{N}=4$ extended supersymmetry coupled to matter. That theory has a scalar potential, which can be related to the gauge-fixing of the Chern-Simons on the supergroup.

Of course, there are several gauge-fixing choices and we will explore them, pointing out the relevant features of the corresponding quantized theories. In the BRST formalism the gauge-fixing is chosen by adding to the action the BRST variation of the gauge-fixing fermion $\Psi$. The latter has to carry negative ghost number, it should be Lorentz invariant and, since at this stage we are gauge-fixing only the fermionic gauge symmetries, it has to be gauge invariant under the bosonic symmetries.

In particular, we point out that, among the possible gauge-fixing choices, there is an unconventional one, whose degrees of freedom correspond to a propagating massive Dirac spinor, which reproduces the field content of the model described by Alvarez, Valenzuela and Zanelli [11, 12], to be referred to in the sequel as AVZ model. The latter is based on an $N=2$ supergroup and provides a phenomenological description of graphene. In that case, a three dimensional Chern-Simons theory with $\operatorname{OSp}(2 \mid 2)$ gauge group, and the fermionic 1 -forms $\psi_{I}^{\alpha}, I=1,2$, in the bifundamental of the $\mathrm{Sp}(2) \times \mathrm{SO}(2)$ group are written in terms of spin- $1 / 2$ fields $\chi_{I}^{\alpha}$ through of the Ansatz:

$$
\psi_{I}^{\alpha}=i e^{i}\left(\gamma_{i}\right)^{\alpha}{ }_{\beta} \chi_{I}^{\beta},
$$

where $e^{i}, i=0,1,2$, are the vielbein 1 -forms of the three dimensional spacetime and $\gamma^{i}$ are the corresponding gamma matrices. Since $e^{i}$ and $\chi_{I}^{\beta}$ only enter the action through the above Ansatz, the theory is invariant under the local rescaling symmetry [11]: $e^{i} \rightarrow$ $\lambda(x) e^{i}, \quad \chi_{I}^{\beta} \rightarrow \frac{1}{\lambda(x)} \chi_{I}^{\beta}, \lambda(x)$ being a real function. The $\operatorname{Sp}(2)$-connection is identified with 
the Lorentz one $\omega^{a b}$, and a space-time torsion $T^{i}=\mathcal{D} e^{i}$ is allowed for. By suitably fixing the local rescaling symmetry of $e^{i}, T^{i}$ can be made constant of the form:

$$
T^{i}=\mathcal{D} e^{i} \equiv d e^{i}+\omega^{i}{ }_{j} \wedge e^{j}=\tau \epsilon^{i}{ }_{j k} e^{j} \wedge e^{k},
$$

$\tau$ being a dimensionful constant.

The Ansatz (1.2) amounts to setting the spin-3/2 component of the gravitino fields to zero, keeping however a non-zero spin- $1 / 2$ component. As a consequence of this choice, the original Chern-Simons theory yields an effective model describing a propagating massive spin-1/2 Dirac field $\chi^{\alpha}=\chi_{1}^{\alpha}+i \chi_{2}^{\alpha}$, whose mass is related to the spacetime torsion $\tau$. This model is suited to describe graphene in the presence of space-time curvature and torsion.

In [13] the model of [11] is embedded in supergravity. First of all it is embedded in $A d S_{3}$ supergravity by identifying its gauge supergroup with the $\operatorname{OSp}(2 \mid 2)$-factor of the super- $A d S_{3}$ symmetry $\operatorname{OSp}(2 \mid 2) \times \mathrm{SO}(2,1)$. The $D=3$ supergravity is then characterized as the boundary theory of an $A d S_{4}$ supergravity with $N=2$ supersymmetry [14]. In this holographic correspondence, an appropriate parametrization of the $A d S_{4}$ space is chosen, which corresponds to an $A d S_{3}$-slicing of the same space. Furthermore, by choosing suitable boundary conditions for the four-dimensional fields, the model of [11] is retrieved at the $A d S_{3}$ boundary. In this picture the spin-1/2 field $\chi^{\alpha}$, which ought to describe the collective electron modes in the graphene, originates from the radial component of the $D=4$ gravitino field (i.e. the component of the gravitino 1-form along the direction perpendicular to the boundary), and the torsion parameter $\tau$, which behaves as a mass term for the spinor $\chi$, is naturally related to the curvature of the $A d S_{3}$ spacetime. However, while in the AVZ model of $[11,12]$ the presence of a cosmological constant, with the corresponding enhancement of the gauge symmetry to $\operatorname{OSp}(2 \mid 2) \times \mathrm{SO}(1,2)$, is optional, this is not the case if one aims to identify the $\mathrm{CS}$ model with $\mathrm{D}=3$ supergravity, since the Achucarro-Townsend map [9], on which the identification in [13] is based, requires a non-vanishing cosmological constant, which induces a non-vanishing mass term for the Dirac spinor $\chi$.

In both the constructions in [11] and [13], the condition (1.2) is put by hand. An important goal of the present paper is to retrieve it dynamically within a covariant BRSTquantized setting. As said above, by a Landau-type gauge-fixing of the (gauge) supersymmetry, we are able to see that there is one massless Dirac spinor propagating. However, to compare it with the Ansatz (1.2) for the case of a massive spinor, as it is the case in [13], we have to modify the gauge-fixing term by adding a first order differential and a vorticity term to the action. By a simple analysis of the quadratic part of the action, it is then easy to show that the Dirac spinor has a non-vanishing mass related to the cosmological constant.

Another outcome of our analysis is the study of the symmetry properties of the quantum model on the lower-side of the relation in (1.1). In particular we find, at least for the conventional Landau gauge-fixing, that the quantum CS-theory exhibits a rigid $\mathcal{N}=4$ world-volume supersymmetry on $A d S_{3}$, which comprises, besides the BRST symmetry, also an emerging "vector"-supersymmetry [15-18]. We shall expand on this particular issue in a forthcoming paper [19].

The paper is organized as follows: in section 2 we define the classical symmetries of the $D=3$ Chern-Simons supergravity and its BRST symmetries. In particular in 
subsection 2.1 we recall the basic facts about three dimensional AdS $N$-extended supergravity. In subsection 2.2 we review its interpretation as describing the graphene in the AVZ Ansatz. In subsection 2.3, we present the BRST formulation. In subsections 2.4 and 2.5, the secondary BRST symmetry $\bar{s}$ is discussed as well as the "vector" BRST symmetry transformations $s_{\mu}, \bar{s}_{\mu}$. In section 3 , we finally quantize the model by considering different types of gauge-fixing: 1) a conventional gauge-fixing leading to a massless Dirac spinor (Landau gauge-fixing) and yielding a dual $\mathcal{N}=4$ supersymmetric model, 2) Landau gauge-fixing with additional non-linear terms to reproduce the scalar potential in the dual theory, 3) an additional term (Nakanishi-Lautrup term) to allow for mass deformations of the model, 4) an $s \bar{s}$ gauge-fixing based on the presence of a secondary BRST symmetry $\bar{s}$ and, finally, 5) an unconventional gauge-fixing, which reproduces the AVZ Ansatz with a non-vanishing mass.

We conclude with the summary and with the future perspectives.

\section{$2 \mathrm{D}=3 N$-extended Chern-Simons supergravity}

\subsection{Basic facts}

We first recall some basic facts about $D=3$ supergravity with negative cosmological constant. As is well-known from the work of Achucarro and Townsend [9], $N$-extended $\mathrm{D}=3$ supergravity on $A d S_{3}$ can be formulated in terms of a Chern-Simons gauge theory. More precisely, in the absence of non-trivial boundary conditions, it can be rephrased as the difference of two Chern-Simons Lagrangians, associated, respectively, with the supergroups $G_{+}=\operatorname{OSp}(p \mid 2)$ and $G_{-}=\operatorname{OSp}(q \mid 2)$, with $p+q=N$ (and bosonic parts $\mathrm{SO}(p) \times \operatorname{Sp}(2)_{(+)}$ and $\mathrm{SO}(q) \times \mathrm{Sp}(2)_{(-)}$, respectively):

$$
\mathcal{L}_{\mathrm{SUGRA}}=\mathcal{L}_{\mathrm{CS}}^{\left(G_{+}\right)}-\mathcal{L}_{\mathrm{CS}}^{\left(G_{-}\right)}
$$

The gauge connections of the two CS theories are

$$
\begin{aligned}
& \mathcal{A}_{(+)}=\frac{1}{2} \omega_{(+)}^{\imath \jmath} \mathbb{J}_{\imath \jmath}+A_{(+)}^{I J} \mathbb{T}_{I J}+\overline{\mathbb{Q}}^{\alpha \mid I} \psi_{\alpha \mid I}^{(+)} \\
& \mathcal{A}_{(-)}=\frac{1}{2} \omega_{(-)}^{\hat{\imath} \hat{\jmath}} \mathbb{J}_{\hat{\imath} \hat{\jmath}}+A_{(-)}^{\dot{I} j} \mathbb{T}_{\dot{I} j}+\overline{\mathbb{Q}}^{\dot{\alpha} \mid \dot{I}} \psi_{\dot{\alpha} \mid \dot{I}}^{(-)} .
\end{aligned}
$$

Here $\mathbb{J}_{\imath \jmath}(\imath, \jmath=0,1,2), \mathbb{J}_{\hat{\imath} \hat{\jmath}}(\hat{\imath}, \hat{\jmath}=0,1,2)$ are the generators of $\mathfrak{s o}(1,2)_{(+)} \sim \mathfrak{s p}(2)_{(+)}$and $\mathfrak{s o}(1,2)_{(-)} \sim \mathfrak{s p}(2)_{(-)}$, respectively, $\mathbb{T}_{I J}(I, J=1, \cdots p), \mathbb{T}_{\dot{I} j}(\dot{I}, \dot{J}=1, \cdots q)$ are the generators of $\mathrm{SO}(p)$ and $\mathrm{SO}(q)$, respectively, while $\mathbb{Q}^{\alpha \mid I}, \mathbb{Q}^{\dot{\alpha} \mid \dot{I}}\left(\alpha=1,2 \in \mathrm{Sp}(2)_{(+)}, \dot{\alpha}=\right.$ $\left.1,2 \in \mathrm{Sp}(2)_{(-)}\right)$are the (Majorana) fermionic generators of the two supergroups. ${ }^{1}$ Finally, $\omega_{( \pm)}, A_{( \pm)}, \psi_{( \pm)}$denote the corresponding gauge connections, the last being associated with Majorana spinor 1-forms.

The relation between the topological CS theory and $\mathrm{D}=3 \mathrm{~N}$-extended supergravity (which does not have local propagating degrees of freedom) with $A d S_{3}$ radius $\ell$, is found

\footnotetext{
${ }^{1}$ We denote with a bar the adjoint fermion: $\overline{\mathbb{Q}} \equiv \mathbb{Q}^{t} C, C$ being the charge conjugation matrix.
} 
by introducing the fields:

$$
\begin{aligned}
\omega^{i j} & =\frac{1}{2}\left(\omega_{(+)}^{\imath \jmath}+\omega_{(-)}^{\hat{\imath} \hat{\jmath}}\right) \\
E^{k} & =\frac{\ell}{4}\left(\omega_{(+) \imath \jmath}-\omega_{(-) \hat{\imath} \jmath}\right) \epsilon^{i j k}
\end{aligned}
$$

where $\omega^{i j}$ is identified with the (torsionless) spin connection of the Lorentz algebra:

$$
\mathfrak{s o}(1,2)_{D} \subset \mathfrak{s o}(2,2)=\mathfrak{s o}(1,2)_{(+)} \times \mathfrak{s o}(1,2)_{(-)}
$$

and $E^{i}$ as the bosonic components of the supervielbein of the $N$-extended superspace. Note that, in eqs. (2.4) and (2.5), the identification of the indices $\imath, \jmath, \ldots$ and $\hat{\imath}, \hat{\jmath}, \ldots$ with the anholonomic Lorentz indices $i, j, \ldots$ is understood in the definition of the spin connection and dreibein of $D=3$ supergravity. This corresponds to the fact that the supergravity Lagrangian exhibits manifest invariance with respect to the diagonal Lorentz group $\mathrm{SO}(1,2)_{D} \subset \mathrm{SO}(2,2)$.

Recently, some of us reconsidered, in [13], the Achucarro-Townsend theory [9] and the correspondence (2.1) for the special case $N=p=2, q=0$. In particular, the field equations of the $N=2 A d S_{3}$ supergravity were found as asymptotic boundary conditions on the supergravity field-strengths of $N=2 A d S_{4}$ pure supergravity, along the lines discussed in [14]. In that framework, $\mathrm{OSp}(2 \mid 4)$-invariant Neumann conditions are recovered on the boundary as consistency conditions for supersymmetry of the full action. An $N=p=2 A d S_{3}$ description of those Neumann conditions was found, in [13], for an asymptotic boundary located at $r \rightarrow \infty$ as a particular asymptotic limit, inspired by the so-called "ultraspinning limit" [20] in the Fefferman-Graham parametrization of the $D=4$ superfields.

The resulting $N=p=2, D=3$ supergravity Lagrangian reads:

$$
\mathcal{L}_{(3)}=\left(\mathcal{R}^{i j}-\frac{1}{3 \ell^{2}} E^{i} E^{j}-\frac{1}{2 \ell} \bar{\psi}_{I} \gamma^{i j} \psi_{I}\right) E^{k} \epsilon_{i j k}-\frac{1}{2 \ell} A d A+2 \bar{\psi}_{I}\left(\mathcal{D} \psi_{I}-\frac{1}{2 \ell} \epsilon_{I J} A \psi_{J}\right)
$$

where $i, j, \ldots=0,1,2 \in \mathrm{SO}(1,2)_{D} \subset \mathrm{SO}(2,2), I, J=1,2 \in \mathrm{SO}(2)$. (The $\mathrm{SO}(2)$ repeated indices are meant to be summed over, independently of their position). Its equations of motion are easily written, using $(2.4),(2.5)$, as the $\mathrm{OSp}(2 \mid 2)_{(+)} \times \mathrm{SO}(1,2)_{(-)}$Maurer-Cartan equations:

$$
\begin{aligned}
\mathcal{R}_{(+)}^{\imath \jmath} & =\frac{\mathrm{i}}{\ell} \bar{\psi}_{I} \wedge \gamma_{k} \psi_{I} \epsilon^{\imath \jmath k}, \\
\mathcal{D}_{(+)} \psi_{I} & =\frac{1}{2 \ell} A \wedge \epsilon_{I J} \psi_{J}, \\
d A & =\epsilon_{I J} \bar{\psi}_{I} \wedge \psi_{J}, \\
\mathcal{R}_{(-)}^{\hat{\imath}} & =0,
\end{aligned}
$$

where:

$$
R_{(+)}^{\imath \jmath} \equiv d \omega_{(+)}^{\imath \jmath}+\omega_{(+)}^{\imath k} \wedge \omega_{(+) k}{ }^{\jmath}, R_{(-)}^{\hat{\imath} \hat{\jmath}} \equiv d \omega_{(-)}^{\hat{\imath} \hat{\jmath}}+\omega_{(-)}^{\hat{\imath} \hat{k}} \wedge \omega_{(-) \hat{k}}^{\hat{\jmath}}, \quad A_{(+)}^{I J}=\epsilon^{I J} A
$$




\subsection{A model for graphene from $\mathrm{D}=3$ supergravity}

The aim of the analysis in [13] was to make contact with the results of [11], where the $D=3$ Chern-Simons theory of the supergroup $\operatorname{OSp}(2 \mid 2)_{(+)}$was considered, assuming however a peculiar Ansatz for the odd component of the gauge connection 1-form:

$$
\psi_{I}^{\alpha}=\mathrm{i}\left(\gamma_{i}\right)_{\beta}^{\alpha} \chi_{I}^{\beta} e^{i}
$$

Here, $e^{i}, \gamma^{i}$ are, respectively, the dreibein and a set of gamma matrices on the $D=3$ worldvolume where the Chern-Simons theory is defined. With the assumption (2.9), the CS Lagrangian turns out to describe the local dynamics of the spin- $1 / 2$ field $\chi \equiv \chi_{I=1}+\mathrm{i} \chi_{I=2}$. More precisely, $\chi$ is a Dirac spinor satisfying in general the massive Dirac equation, with mass given in terms of the contorsion $\tau=\frac{1}{6} \epsilon^{i j k}\left(D e_{[i}\right)_{j k]}$. For non-zero $\tau$, as discussed in [11], the contorsion can be set to zero by a redefinition of the spin connection, and with that choice the background space-time turns out to be $A d S_{3}$, with cosmological constant $-\tau^{2}$, and the world-volume symmetry is enhanced to $\mathrm{SO}(2,2)^{\prime} .^{2}$

In [13] it was shown that, in the case of contorsion $\tau=-\frac{1}{\ell}$, the model of [11] can be recovered at the asymptotic boundary of $A d S_{4}, N=2$ supergravity. It corresponds to imposing in a non-trivial way the condition that in $D=4$ supergravity projects out the spin- $1 / 2$ part of the gravitino field:

$$
\Gamma^{\hat{\mu}} \Psi_{\hat{\mu} I}=0 \Rightarrow \gamma^{\mu} \psi_{\mu I}=-\gamma^{r} \psi_{r I}=3 \mathrm{i} \chi_{I} \neq 0
$$

where $\Gamma, \Psi$ denote $D=4$ gamma-matrices and gravitino, respectively, $\hat{\mu}=(\mu, r)=0,1,2,3$ being holonomic world indices.

The Ansatz (2.9) of [11], in light of its relation with supergravity in $D=3$ [9] and in $D=4[13]$, is remarkable in several respects:

- It introduces in the topological Chern-Simons Lagrangian a dependence on the spacetime background and a local dynamics for the spinor $\chi$.

- It implies that the radial component of the $D=4$ gravitino is not suppresed in the asymptotic limit.

- It also implies a non-trivial relation between the world-volume dreibein $e^{i}$ and the bosonic part of the super-dreibein $E^{i}$, which is discussed in detail in [13].

When writing the Ansatz (2.9), a clear distinction has to be made between the target space quantities, which are connections of the superalgebra, and the world-volume quantities $e^{i}$, $\chi$, and the world-volume Lorentz connection. It is appropriate, therefore, to introduce a different notation for the spinorial index on the world-volume, denoting it by "primed" greek letters. Accordingly, eq. (2.9) will then be written, in the following, as:

$$
\psi_{I}^{\alpha}=\mathrm{i}\left(\gamma_{i}\right)_{\beta^{\prime}}^{\alpha} \chi_{I}^{\beta^{\prime}} e^{i} .
$$

\footnotetext{
${ }^{2}$ Here and in the following, we shall distinguish by a prime quantities referred to the world-volume from the analogous quantities on the target space.
} 
In this expression it is manifest that $\left(\gamma_{i}\right)_{\beta^{\prime}}^{\alpha}$ is an intertwiner between the spinor representation of the target space, labeled by $\alpha$, and the one on the world-volume, labeled by $\beta^{\prime}$. An identification between the Lorentz groups on the target space and on the worldvolume is implicit. In flat space, this identification would be unambiguous. However, in the Achucarro-Townsend model a negative cosmological constant is present on the target space, and this naturally induces the same anti-de Sitter geometry also on the worldvolume. This allows multiple choices for the identification of the two Lorentz groups inside the two $\mathrm{SO}(2,2) A d S_{3}$ symmetry groups, which, due to the non trivial relationship between the $E^{i}$ and $e^{i}$, are distinct.

We can identify the Lorentz group, both in the target space and in the worldvolume, with the diagonal $\mathrm{SL}(2)_{D} \subset \mathrm{SO}(2,2)$, which is associated with a Riemannian spin-connection. Alternatively, we can identify the common Lorentz group with one of the two $\mathrm{SL}(2)_{ \pm}$factors. The corresponding spin-connection is torsionful; this is the choice made in [13]. In this latter case, the $\mathrm{SL}(2) \subset \mathrm{SO}(2,2)$ factor which is not identified with the Lorentz group can be interpreted as an internal symmetry, associated with new spinorial indices: $\dot{\alpha}$ in the target space and $\dot{\alpha}^{\prime}$ on the world-volume. This observation will be relevant for the discussion in the next sections.

In the forthcoming section, we will show that the condition (2.9), with all its peculiar properties discussed above, can be naturally reproduced as a (non-standard) gauge-fixing of the gauged supersymmetry of the Chern-Simons theory, or equivalently, in light of the correspondence in (2.1), of $D=3$ supergravity. In the following we are going to reformulate the theory in a BRST covariant framework in order to set up the gauge-fixing properly.

\subsection{BRST formulation of $N=2 A d S_{3}$ supergravity}

In the following we will find useful to keep manifest only the Lorentz subalgebra $\mathfrak{s p}(2)_{D} \sim$ $\mathfrak{s o}(1,2)_{D} \subset \mathfrak{s o}(1,2)_{(+)} \times \mathfrak{s o}(1,2)_{(-)}$, as discussed above. To this aim we introduce the $\mathfrak{s o}(1,2)_{D}$-covariant notations for the $\mathfrak{s o}(1,2)_{(+)} \times \mathfrak{s o}(1,2)_{(-)}$spin connections:

$$
\omega_{ \pm}^{\alpha \beta} \equiv \frac{1}{2} \gamma_{i j}^{\alpha \beta}\left(\omega^{i j} \pm \frac{1}{\ell} E_{k} \epsilon^{i j k}\right)
$$

and for the corresponding field strengths $\mathcal{R}_{ \pm}^{\alpha \beta}=d \omega_{ \pm}^{\alpha \beta}-\frac{1}{2} \omega_{ \pm}^{\alpha \gamma} \epsilon_{\gamma \delta} \wedge \omega_{ \pm}^{\delta \beta}$. From now on the identification of the spinor indices $\alpha=\dot{\alpha}=1,2 \in \operatorname{Sp}(2)_{D}$ is understood. In addition, we rescale the fields as follows: $\psi \rightarrow \sqrt{\frac{\ell}{2}} \psi$ and $A \rightarrow \ell A$.

The equations of motion then read:

$$
\begin{aligned}
& \mathcal{R}_{+}^{\alpha \beta}=-i \psi^{\alpha I} \wedge \psi_{I}^{\beta}, \quad(\mathcal{D} \psi)_{I}^{\alpha}=\frac{1}{2} A \wedge \epsilon_{I J} \psi_{J}^{\alpha}, \quad d A=-i \frac{1}{2} \epsilon^{I J} \epsilon_{\alpha \beta} \psi_{I}^{\alpha} \wedge \psi_{J}^{\beta}, \\
& \mathcal{R}_{-}^{\alpha \beta}=0,
\end{aligned}
$$

where the Lorentz covariant derivative is $\mathcal{D} \psi_{I}^{\alpha}=d \psi_{I}^{\alpha}+\frac{1}{2}\left(\omega_{+}\right)_{\beta}^{\alpha} \psi_{I}^{\beta}$.

\footnotetext{
${ }^{3}$ The $\mathrm{SO}(2)$ indices $I, J, \ldots$ are lowered and raised with a Kronecker delta, which is generally omitted. We always assume that repeated indices are summed over, independently of their position. As far as doublet $\mathrm{SL}(2, \mathbb{C})$-indices are concerned, they are raised and lowered by the $\epsilon$ symbol, using the "NE-SW" convention: $\xi^{\alpha}=\xi_{\beta} \epsilon^{\beta \alpha}, \xi_{\alpha}=\epsilon_{\alpha \beta} \xi^{\beta}$. As for the signature of space-time metric, we use mostly minus convention, as in [13]. Finally for the conjugation of Grassmann numbers we use the convention: $(\xi \lambda)^{*}=\lambda^{*} \xi^{*}$.
} 
The supersymmetry transformations can be cast in the following way

$$
\begin{aligned}
\delta \omega_{-}^{\alpha \beta} & =0, \\
\delta \omega_{+}^{\alpha \beta} & =-2 i \epsilon^{(\alpha I} \psi_{I}^{\beta)}, \\
\delta A & =-i \epsilon^{I J} \epsilon_{\alpha \beta} \epsilon_{I}^{\alpha} \psi_{J}^{\beta}, \\
\delta \psi_{I}^{\alpha} & =\mathcal{D} \epsilon_{I}^{\alpha}-\frac{1}{2} A \epsilon_{I J} \epsilon_{J}^{\alpha} \equiv \nabla \epsilon_{I}^{\alpha} .
\end{aligned}
$$

The supersymmetry parameter $\epsilon_{I}^{\alpha}$ is a local fermionic real parameter. Although we are interested in the quantization of the full gauge symmetry, which requires the gauge-fixing of the full superalgebra, in the present paper we only focus on the generators associated with the supercharges. The corresponding ghosts will be interpreted as scalar fields in the dual picture. The gauge-fixing of the rest of the gauge symmetry is performed along the conventional procedure.

If we promote the local supersymmetry parameter $\epsilon_{I}^{\alpha}$ to a quantum field, it becomes a ghost field that we denote by $\phi_{I}^{\alpha}$. Note that, since $\phi_{I}^{\alpha}$ is the ghost field of the supersymmetry, it has opposite statistics and, therefore, it is a commuting scalar field. On the other hand, it carries a positive ghost charge with respect to a corresponding $\mathrm{U}(1)$ group. Then, $\phi_{I}^{\alpha}$ is intrinsically complex, but appears only holomorphically in the action.

We can translate (2.14) into BRST transformation rules:

$$
\begin{aligned}
s \omega_{-}^{\alpha \beta} & =0, \\
s \omega_{+}^{\alpha \beta} & =-2 i \phi^{(\alpha I} \psi_{I}^{\beta)}, \\
s A & =-i \epsilon^{I J} \epsilon_{\alpha \beta} \phi_{I}^{\alpha} \psi_{J}^{\beta}, \\
s \psi_{I}^{\alpha} & =\mathcal{D} \phi_{I}^{\alpha}-\frac{1}{2} A \epsilon_{I J} \phi_{J}^{\alpha} \equiv \nabla \phi_{I}^{\alpha}, \\
s \phi_{I}^{\alpha} & =0,
\end{aligned}
$$

where we set the BRST transform of the ghost field $\phi_{I}^{\alpha}$ to zero, since we are only dealing with fermionic gauge symmetries.

Let us also check the nilpotency of the BRST transformations. By introducing the two composite fields (one for each bosonic generator of the supergroup, namely $\operatorname{Sp}(2)$ and $\mathrm{SO}(2))$

$$
\mu_{+}^{(\alpha \beta)}=-\phi_{I}^{\alpha} \phi_{I}^{\beta}, \quad \mu_{+[I J]}=-\epsilon_{\alpha \beta} \phi_{I}^{\alpha} \phi_{J}^{\beta}=\epsilon_{I J} \mu_{+},
$$

(the notation is adopted from [2] where $\mu_{+}^{(\alpha \beta)}$ and $\mu_{+}$denote the holomorphic moment maps of the action of the gauge group $\operatorname{Sp}(2) \times \mathrm{SO}(2)$ on the vector space of the ghost fields $\phi_{I}^{\alpha}$ ) we find

$$
\begin{aligned}
s^{2} \omega_{-}^{\alpha \beta} & =0, \\
s^{2} \omega_{+}^{\alpha \beta} & =i \nabla \mu_{+}^{(\alpha \beta)}, \\
s^{2} A & =i \nabla \mu_{+}, \\
s^{2} \psi_{I}^{\alpha} & =\frac{i}{2} \mu_{I J}^{+} \psi_{J}^{\alpha}+\frac{i}{2} \mu^{+\mid(\alpha \beta)} \epsilon_{\beta \gamma} \psi_{I}^{\gamma}, \\
s^{2} \phi_{I}^{\alpha} & =0 .
\end{aligned}
$$


Note that the above BRST transformations are not nilpotent (except those on $\omega_{-}$and on $\phi_{I}^{\alpha}$ ), but they yield bosonic gauge transformations of the Lie algebra $\mathfrak{o s p}(2 \mid 2)_{(+)}$with local parameters $-i \mu_{+}^{(\alpha \beta)}$ and $-i \mu_{+}$. A nilpotent BRST symmetry is attainable by adding the ghosts $c^{(\alpha \beta)}$ and $c$ of the bosonic symmetry $\mathrm{Sp}(2) \times \mathrm{SO}(2)$. That follows the conventional procedure and we refer to the vast literature on the subject, see for instance [2]. For the purpose of the present paper, we do not need to describe this sector and therefore we omit it.

To set up the gauge-fixing, one needs also some BRST doublets ${ }^{4}$ which transform in the conjugate representation with respect to $\psi_{I}^{\alpha}$ and $\phi_{I}^{\alpha}$. To this aim we introduce the set $\left(\bar{\phi}_{\alpha}^{I}, \bar{\eta}_{\alpha}^{I}\right),{ }^{5}$ with the BRST tranformations:

$$
s \bar{\phi}_{\alpha}^{I}=\bar{\eta}_{\alpha}^{I}, \quad s \bar{\eta}_{\alpha}^{I}=\frac{i}{2}\left(\mu_{I J}^{+} \bar{\phi}_{\alpha J}+\mu^{+\mid(\delta \beta)} \epsilon_{\alpha \delta} \bar{\phi}_{\beta I}\right) .
$$

One can verify that, acting twice with the BRST differential $s$ on the latter fields, one has again nilpotency up to gauge transformations (as in eq. (2.17)). With the anti-ghost fields $\bar{\phi}_{\alpha}^{I}$, we can define the moment maps related to the Kähler structure $K=d \phi_{I}^{\alpha} \wedge d \bar{\phi}_{\alpha}^{I}$ as follows:

$$
\mu_{3}^{\alpha \beta}=-\bar{\phi}_{I}^{(\alpha} \phi_{I}^{\beta)}, \quad \mu_{3 \mid I J}=-\phi_{[I}^{\alpha} \bar{\phi}_{J]}^{\beta} \epsilon_{\alpha \beta},
$$

where, again, the notation is adopted form [2]. In addition to the holomorphic moment maps given in (2.16), we can also introduce the anti-holomorphic moment maps:

$$
\mu_{-}^{(\alpha \beta)}=-\bar{\phi}_{I}^{\alpha} \bar{\phi}_{I}^{\beta}, \quad \mu_{-\mid[I J]}=-\epsilon_{\alpha \beta} \bar{\phi}_{I}^{\alpha} \bar{\phi}_{J}^{\beta}=\epsilon_{I J} \mu_{-} .
$$

There is a hyper-Kähler structure underlying the above relations (2.16), (2.19), (2.20). Indeed, the scalar bosonic ghost fields $\phi$ and $\bar{\phi}$ introduced for the gauge-fixing have a natural interpretation as coordinates on a hyper-Kälher manifold, as emphasized in [2], where the $\mathrm{SU}(2)$ symmetry associated with the hyper-Kähler structure can be made manifest by arranging $\phi_{I}^{\alpha}$ and $\bar{\phi}_{\alpha}^{I}$ in the following doublet $\Phi_{I}^{\alpha \mid A}, A=1,2$ :

$$
\Phi_{I}^{\alpha \mid 1}=\phi_{I}^{\alpha}, \quad \Phi_{I}^{\alpha \mid 2}=-\epsilon^{\alpha \beta} \bar{\phi}_{\beta I} .
$$

Here $A$ labels the eigenvectors of the $\mathrm{U}(1)$ generator having eigenvalues $\pm i$ on the ghost and anti-ghost field, respectively. The hyper-Kähler structure is described by the following triplet of closed 3-forms $\Omega^{A B}=\Omega^{B A}$ :

$$
\Omega^{A B}=\epsilon_{\alpha \beta} d \Phi_{I}^{\alpha A} \wedge d \Phi_{I}^{\beta B} .
$$

We shall also denote by $\Omega=\Omega^{11}=\epsilon_{\alpha \beta} d \phi_{I}^{\alpha} \wedge d \phi_{I}^{\beta}$ and $\bar{\Omega}=\Omega^{22}=\epsilon_{\alpha \beta} d \bar{\phi}_{I}^{\alpha} \wedge d \bar{\phi}_{I}^{\beta}$ the holomorphic and anti-holomorphic structures, respectively. The Kähler form $K$ introduced earlier, on the other hand, coincides with the remaining component of $\Omega^{A B}: K=\Omega^{12}$. In terms of $\Omega^{A B}$, the triholomorphic moment maps, associated with the generators of $\mathrm{Sp}(2) \times \mathrm{SO}(2)$ symmetry group, are defined as follows:

$$
\iota_{V^{(\alpha \beta)}} \Omega^{A B}=-d \mu^{A B \mid(\alpha \beta)}, \iota_{V_{I J}} \Omega^{A B}=-d \mu_{I J}^{A B},
$$

\footnotetext{
${ }^{4} \mathrm{~A}$ BRST doublet is cohomologically trivial and this implies that all observables are independent of it.

${ }^{5}$ Beware: the bar over the fields in the BRST-exact sector denotes the anti-ghost sector.
} 
$V^{(\alpha \beta)}, V_{I J}$ being the Killing vectors generating $\mathrm{Sp}(2)$ and $\mathrm{SO}(2)$, respectively. ${ }^{6}$ The explicit form of the triholomorphic moment maps is readily computed to be:

$$
\mu^{A B \mid(\alpha \beta)}=-\Phi_{I}^{(\alpha \mid A} \Phi_{I}^{\beta) B}, \quad \mu_{I J}^{A B}=-\epsilon_{\alpha \beta} \Phi_{[I}^{\alpha \mid A} \Phi_{J]}^{\beta \mid B}=\epsilon_{I J} \mu^{A B} .
$$

In particular, with reference to the above definition, we have the following identifications:

$$
\begin{aligned}
& \mu^{11 \mid(\alpha \beta)}=\mu_{+}^{(\alpha \beta)} ; \quad \mu^{22 \mid(\alpha \beta)}=\epsilon^{\alpha \gamma} \epsilon^{\beta \delta} \mu_{-(\gamma \delta)} ; \quad \mu^{12 \mid(\alpha \beta)}=\mu_{3}^{(\alpha \beta)} ; \\
& \mu^{11}=\mu_{+} ; \quad \mu^{22}=\mu_{-} ; \quad \mu^{12}=\mu_{3} .
\end{aligned}
$$

In addition, the moment maps satisfy the condition: ${ }^{7}$

$$
\mu_{+}^{(\alpha \beta)} \mu_{+\mid(\alpha \beta)}+2 \mu_{+}^{2}=0,
$$

that is crucial for the closure of the superalgebra of $\mathfrak{o s p}(2 \mid 2)$.

The BRST invariant action is:

$$
\mathcal{L}^{(3)}=\mathcal{L}_{+}^{(3)}-\mathcal{L}_{-}^{(3)},
$$

where

$$
\begin{aligned}
\mathcal{L}_{+}^{(3)} & =\frac{1}{2}\left(\omega_{+}^{\alpha \beta} \wedge d \omega_{+, \alpha \beta}-\frac{1}{3} \omega_{+}^{\alpha \alpha^{\prime}} \wedge \omega_{+, \alpha^{\prime} \beta^{\prime}} \wedge \omega_{+}^{\beta^{\prime} \beta}\right)-2 i \epsilon_{\alpha \beta} \psi^{\alpha I} \nabla \psi_{I}^{\beta}-A \wedge d A \\
\nabla \psi_{I}^{\alpha} & =\left(\delta_{\beta}^{\alpha} d+\frac{1}{2} \omega_{\beta}^{\alpha}\right) \psi_{I}^{\beta}-\frac{1}{2} \epsilon_{I J} A \wedge \psi_{J}^{\alpha}, \\
\mathcal{L}_{-}^{(3)} & =\frac{1}{2}\left(\omega_{-}^{\alpha \beta} \wedge d \omega_{-, \alpha \beta}-\frac{1}{3} \epsilon_{\alpha \beta} \omega_{-}^{\alpha \alpha^{\prime}} \wedge \omega_{-, \alpha^{\prime} \beta^{\prime}} \wedge \omega_{-}^{\beta^{\prime} \beta}\right) .
\end{aligned}
$$

The first piece $\mathcal{L}_{+}^{(3)}$ is the Chern-Simons action related to the superalgebra osp $(2 \mid 2)$, while the second piece $\mathcal{L}_{-}^{(3)}$ is related to the bosonic algebra $\mathfrak{s o}(1,2)$.

\subsection{A secondary BRST symmetry}

From [3], trying to understand the origin of the world-volume supersymmetry, we learn that there is a secondary BRST symmetry, that we denote by $\bar{s}$. It is obtained by exchanging the role of the ghost field $\phi_{I}^{\alpha}$ with that of the anti-ghost $\bar{\phi}_{\alpha}^{I}$, as follows:

$$
\begin{aligned}
\bar{s} \omega_{-}^{\alpha \beta} & =0, \\
\bar{s} \omega_{+}^{\alpha \beta} & =-2 i \bar{\phi}_{I}^{(\alpha} \psi_{I}^{\beta)}, \\
\bar{s} A & =-i \epsilon_{\alpha \beta} \epsilon_{I J} \bar{\phi}_{I}^{\alpha} \psi_{J}^{\beta}, \\
\bar{s} \psi_{I}^{\alpha} & =\mathcal{D} \bar{\phi}_{I}^{\alpha}-\frac{1}{2} A \epsilon_{I J} \bar{\phi}_{J}^{\alpha} \equiv \nabla \bar{\phi}_{I}^{\alpha}, \\
\bar{s} \bar{\phi}_{\alpha}^{I} & =0 .
\end{aligned}
$$

\footnotetext{
${ }^{6}$ In our notations:

$\delta_{\mathrm{Sp}(2)} \Phi_{I}^{\gamma A}=\frac{1}{2} \lambda_{(\alpha \beta)}\left(V^{(\alpha \beta)}\right)_{I}^{\gamma A}=\frac{1}{2} \lambda_{(\alpha \beta)} \epsilon^{\gamma(\alpha} \Phi_{I}^{\beta) A}, \quad \delta_{\mathrm{SO}(2)} \Phi_{I}^{\alpha A}=\frac{1}{2} \lambda^{K L}\left(V_{K L}\right)_{I}^{\alpha A}=-\frac{1}{2} \lambda^{K L} \delta_{I[K} \Phi_{L]}^{\alpha A}$.

${ }^{7}$ The symmetric couple of indices $\alpha \beta$ is lowered by the Cartan-Killing metric $k_{(\alpha \beta)(\gamma \delta)}=\epsilon_{\alpha(\gamma} \epsilon_{\delta) \beta}$.
} 
The Chern-Simons action and the fermionic terms are invariant under this BRST symmetry in the same way as they are invariant under the BRST symmetry $s$. Just as in the latter case, the nilpotency of the $\bar{s}$-BRST transformations is satisfied up to gauge transformations, with parameters $-i \mu_{(\alpha \beta)}^{-}$and $-i \mu^{-}$. The analysis is performed along the same lines as in (2.17). The indices in $\mu_{(\alpha \beta)}^{-}$appear in the lower position, but they can be raised by the Cartan-Killing metric of the superalgebra: $\mu_{\alpha \beta}=-\epsilon_{\alpha \gamma} \epsilon_{\beta \delta} \mu^{\gamma \delta}$.

In addition, the $\bar{s}$ transformation of $\phi_{I}^{\alpha}$ is

$$
\bar{s} \phi_{I}^{\alpha}=-\bar{\eta}_{I}^{\alpha}, \quad \bar{s} \bar{\eta}_{I}^{\alpha}=-\frac{i}{2}\left(\mu_{-} \epsilon_{I J} \phi_{J}^{\alpha}+\mu_{-}^{(\alpha \beta)} \epsilon_{\beta \gamma} \phi_{I}^{\gamma}\right) .
$$

Again, by computing the nilpotency of this new BRST differential $\bar{s}$, we see that eqs. (2.29) and (2.30) close on gauge transformations (the supergauge transformations induced by the supergroup $\operatorname{Osp}(2,2))$ with parameters $-i \mu^{-},-i \mu_{(\alpha \beta)}^{-}$. In order to check the nilpotency, the conditions conjugated to those in (2.26) are used.

The two BRST symmetries have to be compatibile. To this aim we need to check the anticommutation relations between them. It is easy to show that we have

$$
\frac{1}{2}(s \bar{s}+\bar{s} s)=-\frac{i}{2} \mu_{3}^{\alpha \beta} \delta_{(\alpha \beta)}-i \mu_{3} \delta,
$$

where $\delta$ and $\delta_{(\alpha \beta)}$ are the generators of the gauge symmetries $\mathrm{SO}(2)$ and $\mathrm{Sp}(2)$ of the supergroup and $\mu_{3}^{(\alpha \beta)}, \mu_{3}$ are the moment maps related to Kähler structure $K$, introduced in (2.19). This means that the anticommutation of the two BRST transformations yields a gauge transformation with parameters $-i \mu_{3}^{\alpha \beta},-i \mu_{3}$, and therefore they anticommute only when acting on gauge-invariant quantities.

\subsection{Vector BRST symmetry}

Before entering into the detail of the gauge fixing, which will be the subject of next section, let us clarify here where the world-volume supersymmetry on the gauge fixed theory comes from. Even though a general discussion of that issue for all possible gauges will deserve a longer work [19], let us observe that, in the BRST-gauge fixing of Chern-Simons theories, supersymmetry emerges in a very interesting way. Indeed, the action depends upon the world-volume metric only through the gauge-fixing term. The latter, as we shall discuss in the next section, is BRST exact and has the general form $\int s \Psi$, where $\Psi$ is the so-called gauge-fixing fermion, namely a fermionic function of the fields which encodes the gauge fixing. Therefore, the world-volume energy-momentum tensor satisfies the equation [15-18]

$$
\frac{\delta S}{\delta g_{\mu \nu}} \equiv T_{\mu \nu}=s \Gamma_{\mu \nu}
$$

where $\Gamma_{\mu \nu}$ is the variation of the gauge-fixing fermion $\Psi$ with respect to the world-volume metric $g_{\mu \nu}$. It can be proven that the conservation of $\Gamma_{\mu \nu}$ (up to a suitable redefinition of $\left.T_{\mu \nu}\right)$ follows from the following equation:

$$
\partial^{\mu} \Gamma_{\mu \nu}=\delta_{\nu} A_{\mu}^{\alpha \beta} \frac{\delta S}{\delta A_{\mu}^{\alpha \beta}}+\delta_{\nu} A_{\mu,[I J]} \frac{\delta S}{\delta A_{\mu,[I J]}}+\delta_{\nu} \psi_{\mu, I}^{\alpha} \frac{\delta S}{\delta \psi_{\mu, I}^{\alpha}}+\delta_{\nu} \bar{\eta}_{\alpha}^{I} \frac{\delta S}{\delta \bar{\eta}_{\alpha}^{I}}+\delta_{\nu} \phi_{I}^{\alpha} \frac{\delta S}{\delta \phi_{I}^{\alpha}}+\delta_{\nu} \bar{\phi}_{\alpha}^{I} \frac{\delta S}{\delta \bar{\phi}_{\alpha}^{I}}
$$


which implies the existence of a rigid vector BRST-symmetry $\delta_{\nu}$. The form of the field variations, whose explicit realization depends on the gauge-fixing considered, can be read off from the various terms $\delta_{\nu} A_{\mu}^{\alpha \beta}, \ldots, \delta_{\nu} \bar{\phi}_{\alpha}^{I}$.

Moreover, a further vector BRST-symmetry transformation $\bar{\delta}_{\mu}$ leaves invariant the gauge-fixed Chern-Simons lagrangian, as it can be checked for the Laundau gaugefixing (3.6), which will be discussed in section 3.2; in fact, it can be rewritten in terms of the $\bar{s}$ as follows $\int s \Psi=\int \bar{s} \bar{\Psi}$ where the ghost $\phi_{I}^{\alpha}$ and the anti-ghost $\bar{\phi}_{I}^{\alpha}$ are exchanged, but this implies the existence of a further vector BRST-symmetry, $\bar{\delta}_{\mu}$. We shall denote by $s_{\mu}, \bar{s}_{\mu}$ the abstract generators of $\delta_{\mu}, \bar{\delta}_{\mu}$, respectively.

All the symmetries are recombined into an $\mathcal{N}=4$ supersymmetry formulation [17], to be compared with the twisted version of the $\mathcal{N}=4$ supersymmetry of the dual model. Indeed, the scalar supersymmetries represented by the BRST operators $s$ and $\bar{s}$ and the vector supersymmetries $s_{\nu}$ and $\bar{s}_{\nu}$ are naturally combined in the $\mathcal{N}=4$ supersymmetry mentioned earlier. We shall elaborate further on this at the end of subect. 3.1.

\section{Gauge-fixing choices}

\subsection{Counting of D.O.F.'s}

Before discussing the gauge-fixing, it is convenient to count the off-shell degrees of freedom. This will clarify the correspondence, outlined in the Introduction, between the AchucarroTownsend model [9] and an $\mathcal{N}=4$ world-volume supersymmetric Chern-Simons theory coupled to matter, analogous to the model discussed in [1]. In the counting, negative d.o.f.'s mean gauge symmetries.

We have the gauge fields $\omega_{ \pm, \mu}^{(\alpha \beta)}$, each of them having $(3 \times 3-3)$ d.o.f.'s, the fermionic gauge fields $\psi_{I, \mu}^{\alpha}$, with $(2 \times 2 \times 3-2 \times 2)$ and the $\mathrm{SO}(2)$ gauge field $A_{\mu}$ with $(3-1)$ d.o.f.'s. We note that, in the supersymmetric sector, the bosonic d.o.f.'s $(6+2)$ match the fermionic ones.

In addition, we note that $\psi_{I, \mu}^{\alpha}$ is a 1-form carrying one index in the $\operatorname{Sp}(2)$ fundamental representation and one index in the $\mathrm{SO}(2) \mathrm{R}$-symmetry vector representation of the bosonic symmetry in the gauge supergroup. Therefore, at first sight it does not have the features of a Rarita-Schwinger field on the world-volume. The interpretation as a gravitino follows from the identification of the subgroup $\mathrm{Sp}(2) \sim \mathrm{SO}(1,2)$ of the gauge supergroup with the world-volume $\mathrm{SO}(1,2)^{\prime}$ Lorentz group.

In the AVZ model [11], the authors introduce a spinor 0-form $\chi_{I}^{\beta^{\prime}}$ as in eq. (2.11), where the index $\beta^{\prime}$ is a truly spinorial index on the world-volume, and $\gamma_{i}^{\alpha \beta^{\prime}}$ are the Dirac matrices which intertwine between the gauge $\mathrm{SO}(1,2)$ group and the world-volume Lorentz $\mathrm{SO}(1,2)_{L}^{\prime}$ group, as discussed in section 2.2. The matrix $e_{\mu}^{i}$ is the $3 \mathrm{~d}$ dreibein, associated with the adjoint representation of the diagonal subgroup $\operatorname{Sp}(2)_{D}^{\prime} \subset \mathrm{SO}(2,2)^{\prime}$ of the worldvolume isometry group.

However, it would be desiderable to derive (2.11) in terms of a gauge symmetry of the model (which actually reduces the off-shell degrees of freedom from 8 down to 4 ). To this end, let us observe that the felds $\psi_{I}^{\alpha}$ are fermionic 1-forms, therefore their components $\psi_{I, \mu}^{\alpha}$ are fermionic d.o.f.'s. In the ghost sector we introduce the Nakanishi-Lautrup fields 
$\bar{\eta}_{\alpha}^{I}$ needed for the gauge-fixing of the fermionic gauge symmetry. The total amount of fermionic degrees of freedom is then $2 \times 2 \times 3$ for $\psi_{I, \mu}^{\alpha},{ }^{8}$ and $2 \times 2$ for $\bar{\eta}_{\alpha}^{I}$.

All the off-shell fermionic d.o.f.'s in our gauge-fixed model can be arranged into a single spinor field of the form $\Lambda_{I, \alpha^{\prime} \beta^{\prime}}^{\alpha}$ given by

$$
\Lambda_{I \alpha^{\prime} \beta^{\prime}}^{\alpha}=i \gamma_{\alpha^{\prime} \beta^{\prime}}^{\mu} \psi_{\mu, I}^{\alpha}-\frac{1}{2} \epsilon_{\alpha^{\prime} \beta^{\prime}} \bar{\eta}_{I}^{\alpha},
$$

where $\alpha^{\prime}$ and $\beta^{\prime}$ both refer to the diagonal world-volume symmetry $\operatorname{Sp}(2)_{D}^{\prime} \subset \operatorname{SO}(2,2)^{\prime}$. This is the analogue in our setting of the topological twist which was shown in [2] to relate the Gaiotto-Witten model [1] with a gauged version of the Rozansky-Witten one [3]. On the other hand, we can perform a different twisting in order to make contact with the AVZ model and to identify its fermionic degrees of freedom in the present context. This is achieved by decomposing $\Lambda_{I \alpha^{\prime} \beta^{\prime}}^{\alpha}$ with respect to the diagonal subgroup of the target space $\operatorname{Sp}(2)$ (which the index $\alpha$ refers to) and of the world-volume $\operatorname{Sp}(2)_{D}^{\prime}$, the fermionic d.o.f.'s $\Lambda_{I, \alpha^{\prime} \beta^{\prime}}^{\alpha}$, so as to obtain

$$
\Lambda_{I \alpha^{\prime} \beta^{\prime}}^{\alpha}=i\left(\gamma^{\mu}\right)_{\alpha^{\prime}}^{\alpha} \chi_{\mu \beta^{\prime}, I}-2 \delta_{\alpha^{\prime}}^{\alpha} \chi_{I \beta^{\prime}} .
$$

On the right hand side the field $\chi_{\mu \beta^{\prime}, I}$ is identified with the world-volume gravitino, while $\chi_{I \beta^{\prime}}$ contains the AVZ fermionic degrees of freedom.

The full world-volume isometry $\mathrm{SO}(2,2)^{\prime}$ can be made manifest by promoting $\Lambda_{I \alpha^{\prime} \beta^{\prime}}^{\alpha}$ to an object in the $(1 / 2,1 / 2,1 / 2)$ of $\operatorname{Sp}(2) \times \operatorname{Sp}(2)_{+}^{\prime} \times \operatorname{Sp}(2)_{-}^{\prime}$ :

$$
\Lambda_{I \alpha^{\prime} \beta^{\prime}}^{\alpha} \longrightarrow \Lambda_{I \alpha^{\prime} \dot{\beta}^{\prime}}^{\alpha}
$$

where we recall that $\alpha^{\prime}$ and $\dot{\beta}^{\prime}$ refer to the groups $\operatorname{Sp}(2)_{+}^{\prime}$ and $\operatorname{Sp}(2)_{-}^{\prime}$, respectively.

The on-shell analysis is difficult since there are no local degrees of freedom. In addition, in the previous sections we introduced the ghost fields $\phi_{I}^{\alpha}, \bar{\phi}_{\alpha}^{I}$, that are scalar bosonic degrees of freedom, which are interpreted as the scalar superpartners of $\Lambda_{I \alpha^{\prime} \beta^{\prime}}^{\alpha}$. As discussed earlier, they naturally parametrise a hyper-Kähler manifold.

We can then group the d.o.f.'s as follows: the gauge fields $\omega_{ \pm}^{(\alpha \beta)}$ and $A$ for the gauge group $\operatorname{Sp}(2) \times \operatorname{Sp}(2) \times \mathrm{SO}(2)$ are described by a Chern-Simons gauge theory, while the other fields $\phi, \bar{\phi}$ and $\Lambda$ build up an $\mathcal{N}=4$ hypermultiplet charged with respect to the gauge fields. The choice of a suitable potential allows to recast the model into a $\mathcal{N}=4$ super-Chern-Simons theory. The corresponding $\mathcal{N}=4$ supersymmetry parameter has the following structure: $\epsilon^{\alpha^{\prime} \dot{\alpha}^{\prime} A}$, where the $\mathrm{SU}(2)$ group acts on the index $A$ [19]. As discussed in section 2, this emerging supersymmetry is related, via the topological twist discussed above, to the scalar and vector BRST quantum symmetries of the model: $s, \bar{s}, s_{\mu}, \bar{s}_{\mu}$. This can be seen by decomposing the corresponding supersymmetry parameters $\epsilon^{\alpha^{\prime} \dot{\alpha}^{\prime}} A$ with respect to $\operatorname{Sp}(2)_{D}^{\prime} \times \mathrm{SU}(2)$ :

$$
\epsilon^{\alpha^{\prime} \dot{\alpha}^{\prime} A}=i\left(\gamma^{i}\right)^{\alpha^{\prime} \dot{\alpha}^{\prime}} \epsilon_{i}^{A}+\epsilon^{\alpha^{\prime} \dot{\alpha}^{\prime}} \epsilon^{A},
$$

where $\epsilon^{A=1}, \epsilon^{A=2}$ correspond to $s$ and $\bar{s}$ BRST symmetries and $\epsilon_{i}^{A=1}, \epsilon_{i}^{A=2}$ correspond to vector supersymmetries $s_{\mu}$ and $\bar{s}_{\mu}$.

\footnotetext{
${ }^{8}$ Indeed, in the BRST-invariant gauge-fixed action, all d.o.f.'s are propagating.
} 


\subsection{Landau gauge-fixing and $\mathcal{N}=4$ supersymmetry}

The gauge-fixed Lagrangian (only for the fermionic gauge symmetry) has the general form

$$
\mathcal{L}=\mathcal{L}^{(3)}+\mathcal{L}^{\text {g.f. }}, \quad \mathcal{L}^{\text {g.f. }}=s\left(\bar{\phi}_{\alpha}^{I} \mathcal{F}_{I}^{\alpha}\left(\omega_{+}, \psi_{I}^{\alpha}, A, \phi_{I}^{\alpha}\right)\right), \quad S=\int_{\mathcal{M}_{3}} \mathcal{L}
$$

where $\Psi=\bar{\phi}_{\alpha}^{I} \mathcal{F}_{I}^{a}\left(\omega_{+}, \psi_{I}^{\alpha}, A, \phi_{I}^{\alpha}\right)$ is known as the gauge-fixing fermion which encodes the gauge-fixing and depends upon the gauge fields $\left(\omega_{+}, \psi_{I}^{\alpha}, A\right)$ and possibly also on the ghost field $\phi_{I}^{\alpha}$.

Let us discuss the gauge-fixing in detail. As already mentioned in the introduction, we do not introduce here the ghosts for the bosonic part of the gauge symmetry, since we are only interested in the gauge-fixing of the odd part of the superalgebra. The gauge-fixing of the even part of the gauge algebra can be done according to the standard procedure.

Let us start with a gauge-fixing, known also as Landau gauge fxing, of the following form (up to total derivatives)

$$
\begin{aligned}
S_{A}=2 \int s\left(\bar{\phi}_{\alpha}^{I} \nabla \star \psi_{I}^{\alpha}\right)= & -2 \int\left(\nabla \phi_{I}^{\alpha} \wedge \star \nabla \bar{\phi}_{\alpha}^{I}+\psi_{I}^{\alpha} \wedge \star \nabla \bar{\eta}_{\alpha}^{I}\right) \\
& +2 \int\left(-i \phi^{J(\alpha} \psi_{J}^{\beta)} \bar{\phi}_{\alpha}^{I} \star \psi_{\beta I}-\frac{1}{2} \epsilon^{K L} \phi_{K}^{\gamma} \psi_{L}^{\delta} \epsilon_{\gamma \delta} \bar{\phi}_{\alpha}^{I} \epsilon_{I}^{J} \star \psi_{J}^{\alpha}\right) .
\end{aligned}
$$

The Hodge dual $\star$ is needed to write the gauge fixed action on any three dimensional manifold, given a metric on it. In particular, we have: $\psi_{I}^{\alpha} \wedge \star \psi_{J}^{\beta}=\psi_{\mu, I}^{\alpha} g^{\mu \nu} \psi_{\nu, J}^{\beta} V^{(3)}$, where $g^{\mu \nu}$ is the inverse metric on the world-volume $\mathcal{M}^{(3)}$, whose volume form is $V^{(3)}=\star 1$. Notice that a world-volume metric is needed only in the gauge-fixing term and it is not present in the gauge-invariant action $\mathcal{L}^{(3)}$.

The first term in (3.6) is an usual kinetic term for complex bosonic fields $\phi_{I}^{\alpha}, \bar{\phi}_{\alpha}^{I}$. The second term contains a first-order linear differential operator on $\bar{\eta}_{\alpha}^{I}$ which, together with the gravitino field equation from eq. (2.28), leads to an invertible wave operator. The last term produces an interaction between $\psi$ and the ghost fields $\phi$ and $\bar{\phi}$. Notice that the ghost fields in the present case are bosonic commuting fields, therefore the kinetic term leads to a positive definite metric for the Hilbert space.

Now, we check that the gauge-fixing fixes the fermionic gauge symmetry and we study the wave operator in the fermionic sector. In order to simplify the discussion, we neglect for the time being the interactions among ghost fields $\left(\phi_{I}^{\alpha}, \bar{\phi}_{\alpha}^{I}\right)$.

The free equations for $\psi_{I}^{\alpha}$ and for $\bar{\eta}_{\alpha}^{I}$ read

$$
2 \epsilon_{\alpha \beta} \nabla \psi_{I}^{\beta}+\star \nabla \bar{\eta}_{I \alpha}=0, \quad \nabla \star \psi_{I}^{\alpha}=0 .
$$

We re-write these equations in components as follows

$$
\epsilon^{\mu \nu \rho} \epsilon_{\alpha \beta} \nabla_{\nu} \psi_{\rho, I}^{\alpha}+\nabla^{\mu} \bar{\eta}_{I \alpha}=0, \quad \nabla^{\mu} \psi_{\mu, I}^{\alpha}=0 .
$$

The second equation is the usual Landau-Lorentz gauge-fixing for the gravitino.

By standard manipulations, using the Clifford algebra on the world-volume and the gauge-fixing condition, we have

$$
2\left(\gamma_{\mu}\right)_{\alpha \beta} \nabla^{\mu}\left(\gamma^{\nu} \psi_{\nu, I}\right)^{\beta}+\left(\gamma_{\mu}\right)_{\alpha}^{\beta} \nabla^{\mu} \bar{\eta}_{\beta}^{I}=\not 2\left(2 \epsilon_{\alpha \beta}\left(\psi_{I}^{\beta}\right)+\bar{\eta}_{\alpha}^{I}\right)=0
$$


form which we get the following combination

$$
\bar{\eta}_{\alpha}^{I}=-2\left(\psi_{I}\right)_{\alpha}+\sigma_{\alpha}^{I}
$$

where $\sigma_{\alpha}^{I}$ is a solution of the massless Dirac equation $\not \partial \sigma_{\alpha}^{I}=0$. However, it is possible to further modify the gauge-fixing in order to introduce a mass term for the Dirac field.

\subsection{Feynman gauge fixing and mass deformations}

The Feynman gauge-fixing also requires the introduction of the Nakanishi-Lautrup auxiliary field $\bar{\eta}^{\alpha}$. It can be added to the gauge-fixing as follows

$$
\begin{aligned}
S_{B} & =2 \int s\left(\beta \bar{\eta}_{\alpha}^{I} \epsilon^{\alpha \beta} \bar{\phi}_{\beta, I}\right) V^{(3)} \\
& =2 \int \beta\left(\bar{\phi}_{\alpha}^{I}\left(\mu_{+, I J}(\phi) \epsilon^{\alpha \beta}+\mu_{+}^{\alpha \beta}(\phi) \delta_{I J}\right) \bar{\phi}_{\beta}^{J}+\bar{\eta}_{\alpha}^{I} \epsilon^{\alpha \beta} \bar{\eta}_{\beta, I}\right) V^{(3)} \\
& =2 \int \beta\left(\mu_{3, \alpha}{ }^{\beta} \mu_{3, \gamma}{ }^{\delta} \epsilon_{\alpha \gamma} \epsilon^{\beta \delta}+\mu_{3, K}^{I} \mu_{3, I}{ }^{K}+\bar{\eta}_{\alpha}^{I} \epsilon^{\alpha \beta} \bar{\eta}_{\beta, I}\right) V^{(3)}
\end{aligned}
$$

Here the first two terms in the last line are potential terms for the bosonic ghost fields $(\phi, \bar{\phi})$, while the third one is a mass term for $\bar{\eta}$. We used the definition of $\mu_{3}$ to simplify the expression and $\beta$ is a gauge-fixing parameter.

The form of the potential generated by the present gauge-fixing is similar to the mass deformations of the Gaiotto-Witten model discussed in [21, 22]. As mentioned in the Introduction, the model we are considering bears resemblance with the ABJM model [10] in that it is described by the difference of two Chern-Simons. However, as is apparent from (3.11), the BRST variation of this gauge-fixing term cannot generate higher powers of $\phi$ and $\bar{\phi}$ which could reproduce the sextic scalar potential of ABJM models.

The gauge-fixing produces a $\bar{\eta}^{2}$ term, which serves as the Nakanishi-Lautrup term, modifying the quadratic part of the $\psi-\bar{\eta}$ system, whose equations of motion were discussed in the previous section.

\subsection{Non-linear Feynman gauge-fixing and Gaiotto-Witten model}

One can add further interaction terms by introducing a more general gauge-fixing piece of the form

$$
\begin{aligned}
S_{B}^{\prime} & =\int s\left(\bar{\eta}_{\alpha}^{I} V_{I J}^{\alpha \beta}(\phi) \bar{\phi}_{\beta}^{J}\right) V^{(3)} \\
& =\int\left(\bar{\phi}_{\alpha}^{K} \bar{\phi}_{\beta}^{J}\left(\mu_{+, K}^{I} V_{I J}^{\alpha \beta}+\mu_{+, \gamma}^{\alpha} V_{I J}^{\gamma \beta}\right)+\bar{\eta}_{\alpha}^{I} V_{I J}^{\alpha \beta} \bar{\eta}_{\beta}^{J}\right) V^{(3)}
\end{aligned}
$$

where $V_{I J}^{\alpha \beta}$ is a generic tensor built out of the $\phi$ 's. As can be noticed, this additional term modifies the action by non-quadratic terms and it is responsible of the generation of the scalar potential. As discussed in [2], a suitable choice of $V_{I J}^{\alpha \beta}(\phi)$ (compatible with the bosonic gauge invariance of the model) leads to the scalar potential of the dual theory and the coupling with the fermions. 


\subsection{Unconventional gauge fixing and AVZ Ansatz}

Let us now add a new term to the equations of motion and recompute the wave operator. In particular, we will derive the AVZ Ansatz for a massive Dirac spinor by a suitable choice of the gauge-fixing parameters. The corresponding quadratic part of the Lagrangian is

$$
\mathcal{L}=2 \epsilon_{\alpha \beta} \epsilon^{\mu \nu \rho} \psi_{\mu, I}^{\alpha} \nabla_{\nu} \psi_{\rho, I}^{\beta}+\bar{\eta}_{\alpha}^{I}\left(\nabla^{\mu} \psi_{\mu, I}^{\alpha}-2 i \beta\left(\gamma^{i}\right)^{\alpha}{ }_{\beta} \psi_{i, I}^{\beta}\right)+2 \beta \epsilon^{\alpha \beta} \bar{\eta}_{\alpha}^{I} \bar{\eta}_{\beta, I}
$$

where the last term is the usual quadratic term in the Nakanishi-Lautrup field $\bar{\eta}_{\alpha}^{I}{ }^{9}$

The new equations of motion are

$$
\begin{aligned}
4 \epsilon^{\mu \nu \rho} \nabla_{\nu} \psi_{\rho, I}^{\alpha}-2 \nabla^{\mu} \bar{\eta}_{I}^{\alpha}+4 i \beta \gamma^{\mu, \alpha}{ }_{\beta} \bar{\eta}_{I}^{\beta} & =0 \\
\nabla^{\mu} \psi_{\mu, I}^{\alpha}-2 i \beta\left(\gamma^{\mu} \psi_{\mu, I}\right)^{\alpha}+2 \beta \epsilon^{\alpha \beta} \bar{\eta}_{\beta}^{I} & =0 .
\end{aligned}
$$

Manipulating those equations as above, we finally find the massive Dirac equation for the combination $\chi_{I}=-\frac{1}{4}\left(i \psi_{I}+\frac{1}{2} \bar{\eta}_{I}\right)$ which should be identified with the AVZ Dirac spinor.

Finally, we can write down the complete unconventional gauge-fixing of the CS action with this additional piece as follows

$$
\begin{aligned}
S_{A}=\int s\left(\psi_{I}^{\alpha} \wedge \star \nabla \bar{\phi}_{\alpha}^{I}+\psi_{I}^{\alpha} \wedge \star e^{a} \gamma_{a, \alpha}^{\beta} \bar{\phi}_{\beta}^{I}\right) \\
=\int\left[\nabla \phi_{I}^{\alpha} \wedge \star \nabla \bar{\phi}_{\alpha}^{I}+\psi_{I}^{\alpha} \wedge \star \nabla \bar{\eta}_{\alpha}^{I}+\psi_{I}^{\alpha} \wedge \star\left(\epsilon_{\alpha \beta} \psi_{K}^{(\beta} \eta^{K L} \phi_{L}^{\gamma)} \bar{\phi}_{\gamma}^{I}+\eta^{I J} \psi_{[J}^{\gamma} \epsilon_{\gamma \beta} \phi_{K]}^{\beta} \bar{\phi}_{\alpha}^{K}\right)\right. \\
\left.\quad+\nabla \phi_{I}^{\alpha} \wedge \star e^{i} \gamma_{i, \alpha}^{\beta} \bar{\phi}_{\beta}^{I}+\psi_{I}^{\alpha} \wedge \star e^{i} \gamma_{i, \alpha}^{\beta} \bar{\eta}_{\beta}^{I}\right] .
\end{aligned}
$$

No additional term is produced by the BRST variation of the second term of the gaugefixing. In addition, no BRST variation is required for the $3 \mathrm{~d}$ vielbein $e^{i}$. The new term leading to the unconventional gauge-fixing and to the identification of our fermionic fields with the AVZ Ansatz modifies the kinetic term of the bosonic ghost fields (viewed as coordinates of a hyper-Kähler manifold) by a vorticity term involving a first order differential.

\section{$3.6 s \bar{s}$-gauge fixing}

Finally, let us come to the last example of gauge-fixing that can be constructed. Given the fact that there are two BRST symmetries, instead of writing the gauge-fixing as the $s$-variation of $\Psi$, one can construct the gauge-fixing as follows

$$
\mathcal{L}^{g \cdot f \cdot}=s \bar{s} \Sigma
$$

where $\Sigma$ has no ghost charge, it is Lorentz invariant and it must also be gauge invariant under the bosonic subgroup of the supergroup $\operatorname{OSp}(2 \mid 2)$. Since $s$ and $\bar{s}$ are nilpotent, the total action $S$ will be $s$ and $\bar{s}$ invariant. Acting with $s$, it is trivially zero, while to check the $\bar{s}$-invariance, we have to anticommute $\bar{s}$ with $s$ and that can be done at the price of

\footnotetext{
${ }^{9}$ In the case of usual gauge symmetry the associated BRST symmetry is $s A=\nabla c, s c=-\frac{1}{2}[c, c], s \bar{c}=$ $b, s b=0$ where $b$ is the Nakanishi-Lautrup field. Then, the gauge-fixing Lagrangian is $s \operatorname{Tr}\left(\bar{c} d \star A+\frac{1}{2} \xi \bar{c} b\right)=$ $\operatorname{Tr}\left(b d \star A+\frac{1}{2} \xi b^{2}\right)-\operatorname{Tr}(\bar{c} d \star \nabla c)$. The equations of motion for $b$ is $b=-\frac{1}{\xi} d \star A$ which implies that gauge-fixing for $A$.
} 
a gauge transformation in the subgroup. However, being $\Sigma$ gauge invariant, this gauge transformation is ineffective and nilpotency of $\bar{s}$ does the rest.

The Landau gauge-fixing plus non-linear terms (3.6)-(3.12) can be written as follows

$$
\mathcal{L}^{g . f .}=s \bar{s}\left(\psi_{I}^{\alpha} \epsilon_{\alpha \beta} \eta^{I J} \wedge \star \psi_{J}^{\beta}+\bar{\eta}_{\alpha}^{I} \epsilon^{\alpha \beta} \bar{\eta}_{\beta}^{I} \star 1\right) .
$$

Notice that those terms are gauge invariant under the bosonic gauge symmetries of the supergroup $\mathrm{SO}(2)$ and $\mathrm{Sp}(2)$ and, therefore, as pointed out earlier, the order of $s$ and $\bar{s}$ in front of the parenthesis is irrelevant. We have made explicit the contraction of the indices.

Using the present framework, we can rewrite the unconventional gauge-fixing in a similar way by observing that since the group $\operatorname{Sp}(2)$ is the spin group associated with $\mathrm{SO}(1,2)$ (the Lorentz group of the world-volume), we can use the intertwiners $\gamma_{i}^{\alpha \beta}$ (or even better $\Gamma_{i \beta}{ }^{\alpha}=i \epsilon_{\alpha \gamma} \gamma_{i}^{\gamma \beta}$ ) to extend the above gauge-fixing term as follows

$$
\mathcal{L}^{g . f .}=s \bar{s}\left(\psi_{I}^{\alpha} \epsilon_{\alpha \beta} \wedge \star \psi_{I}^{\beta}+\bar{\eta}_{\alpha}^{I} \epsilon^{\alpha \beta} \bar{\eta}_{\beta \mid I} \star 1+\beta \psi_{I}^{\alpha} \wedge \star e^{i} \Gamma_{i \alpha}^{\beta} \bar{\eta}_{\beta}^{I}\right)
$$

where $e^{i}$ is the wordvolume vielbein and $\star e^{i}$ is the world-volume Hodge dual and $\beta$ is the gauge-fixing parameter discussed in the previous section. This beautiful structure contains all possibile quadratic terms written in terms of only the fermionic fields of the theory, namely $\psi_{I}^{\alpha}$ and $\bar{\eta}_{\alpha}^{I}$. They are gauge invariant under the bosonic symmetries of the supergroup together with the Lorentz transformations on the flat index of $e^{i}$.

To conclude, we observe that we can add a further term in order to build a quadratic term, of the form

$$
s \bar{s}\left(A^{(\alpha \beta)} \gamma_{i \alpha \beta} \wedge \star e^{i}\right)
$$

which carries zero ghost number and is a 3 -form. It is manifestly Lorentz invariant and its variation gives

$$
s\left(\psi^{\alpha} \wedge \bar{\phi}^{\beta}\left(\gamma_{i}\right)_{\alpha \beta} \star e^{i}\right)=\nabla \phi^{\alpha} \wedge \bar{\phi}^{\beta}\left(\gamma_{i}\right)_{\alpha \beta} \star e^{i}-\psi^{\alpha} \wedge \bar{\eta}^{\beta}\left(\gamma_{i}\right)_{\alpha \beta} \star e^{i} .
$$

The first term is a viscosity term modifying the quadratic part of the action for the scalar fields. The second term is an off-diagonal mass term for the fermions.

Therefore, also in the present formulation, there are at least two supercharges in the game and part of the supersymmetry is preserved. We leave further consideration in a forthcoming more detailed work [19].

\section{Conclusions and outlook}

In the present work, we have considered different ways of quantizing Chern-Simons supergravity revealing important features of the same theory. This is achieved by applying the analysis of Kapustin and Saulina [2] to this particular Chern-Simons theory describing pure $D=3$ supergravity on an $\mathrm{AdS}_{3}$ background.

The novel feature which characterizes our setting is indeed the presence of a curved background with negative cosmological constant. In this framework both the fermionic degrees of freedom of the AVZ model [11] and the topological twist of [2] find a natural 
interpretation, the latter being related to the choice of the Lorentz $\operatorname{Sp}(2)$ world volume spin connection within the $\mathrm{SO}(2,2)$ isometry group of space-time.

Our analysis led us to consider different choices of the gauge fixing of the fermionic symmetries. The resulting models exhibit some amount of supersymmetry, which emerges from the BRST quantization. The ultimate goal, which will be tackled in a forthcoming work [19], is the study of the supersymmetry associated with the different gauge-fixing choices. In this respect let us summarize the results:

1. Landau Gauge Fixing: this is achieved by setting to zero the Nakanishi-Lautrup terms of the gauge-fixing (3.11) and non-linear gauge fixing terms (3.12). The model has a manifest $\mathcal{N}=4$ supersymmetry as a result of a combination of BRST symmetries $(s$ and $\bar{s}$ ) and of the vector supersymmetries.

2. Kapustin-Saulina Gauge Fixing: adding the non-linear terms (3.12). Those terms are needed to reproduce the scalar potential of the dual theory. In the dual picture, the theory still displays an $\mathcal{N}=4$ supersymmetry, though realised in a non-linear way. That supersymmetry is a manifestation of the BRST symmetries of the original Chern-Simons supergravity and of the vector supersymmetris, which however are not evident in the twisted version due to the non-linearities. This leads to an open problem: to show that the CS supergravity with this particular gauge-fixing admits a formulation with a manifest non-linear vector supersymmetry.

3. Gauge Fixing with Mass Terms: in order to reproduce the deformations discussed in [21-23] one has to change the gauge-fixing adding a new Nakanishi-Lautrup term. Its BRST variation leads to the expected terms.

4. Unconventional Gauge Fixing: as shown in the text, the choice of a particular gaugefixing with a vorticity term, upon identification of the world-volume Lorentz group with the $\mathrm{SO}(1,2)$ target gauge symmetry, leads to the AVZ Ansatz and to the interpretation of the gravitino in terms of the graphene fermion. It is to be explored how much of the supersymmetry survives in the present context.

5. $s \bar{s}$ Gauge-Fixing: choosing this form of the gauge-fixing there are less deformations allowed in the Lagrangian; nonetheless both the BRST and the secondary BRST symmetry are present. In addition, the vorticity term discussed in the previous item is introduced by suitable terms combining the gauge field and the world-volume dreibein. It would be very interesting to explore the consequences of this choice.

The present analysis paves the way to further investigation in different directions: the detailed study of the supersymmetric properties of the quantum dual models originating from different gauge-fixings; the generalization of the present analysis to generic $N$-extended $A d S_{3}$ supergravities; the investigation of the holographic $D=4$ duals to these models. As for the latter objective, a natural candidate would be the maximal $D=4$ supergravity realized on the $\mathcal{N}=4 A d S_{4}$ vacuum [24, 25], which describes a class of Type IIB Janus solutions $[26,27]$. 
As a concluding remark: we have shown that the $N$-extended $\mathrm{D}=3$ supergravity can be reformulated in terms of worldvolume supersymmetric models coupled to matter. This might be useful for computation of interesting observables in both sides of this duality. We leave this to further investigations.

\section{Acknowledgments}

We thank L. Castellani, R. D'Auria, P. Fré, R. Olea, S. Penati, D. Seminara and J. Zanelli for useful discussions on several issues of the present work.

Open Access. This article is distributed under the terms of the Creative Commons Attribution License (CC-BY 4.0), which permits any use, distribution and reproduction in any medium, provided the original author(s) and source are credited.

\section{References}

[1] D. Gaiotto and E. Witten, Janus Configurations, Chern-Simons Couplings, And The theta-Angle in $N=4$ Super Yang-Mills Theory, JHEP 06 (2010) 097 [arXiv:0804.2907] [INSPIRE].

[2] A. Kapustin and N. Saulina, Chern-Simons-Rozansky-Witten topological field theory, Nucl. Phys. B 823 (2009) 403 [arXiv:0904.1447] [INSPIRE].

[3] L. Rozansky and E. Witten, HyperKähler geometry and invariants of three manifolds, Selecta Math. 3 (1997) 401 [hep-th/9612216] [INSPIRE].

[4] V. Mikhaylov and E. Witten, Branes And Supergroups, Commun. Math. Phys. 340 (2015) 699 [arXiv: 1410.1175] [INSPIRE].

[5] N. Drukker and D. Trancanelli, A supermatrix model for $N=6$ super Chern-Simons-matter theory, JHEP 02 (2010) 058 [arXiv: 0912.3006] [INSPIRE].

[6] M. Mariño, Lectures on localization and matrix models in supersymmetric Chern-Simons-matter theories, J. Phys. A 44 (2011) 463001 [arXiv:1104.0783] [InSPIRE].

[7] A. Mauri, H. Ouyang, S. Penati, J.-B. Wu and J. Zhang, BPS Wilson loops in $\mathcal{N} \geq 2$ superconformal Chern-Simons-matter theories, JHEP 11 (2018) 145 [arXiv:1808.01397] [INSPIRE].

[8] M.S. Bianchi, L. Griguolo, A. Mauri, S. Penati and D. Seminara, A matrix model for the latitude Wilson loop in ABJM theory, JHEP 08 (2018) 060 [arXiv: 1802.07742] [INSPIRE].

[9] A. Achucarro and P.K. Townsend, A Chern-Simons Action for Three-Dimensional anti-de Sitter Supergravity Theories, Phys. Lett. B 180 (1986) 89 [InSPIRE].

[10] O. Aharony, O. Bergman, D.L. Jafferis and J. Maldacena, $N=6$ superconformal Chern-Simons-matter theories, M2-branes and their gravity duals, JHEP 10 (2008) 091 [arXiv: 0806.1218] [INSPIRE].

[11] P.D. Alvarez, M. Valenzuela and J. Zanelli, Supersymmetry of a different kind, JHEP 04 (2012) 058 [arXiv:1109.3944] [INSPIRE].

[12] A. Guevara, P. Pais and J. Zanelli, Dynamical Contents of Unconventional Supersymmetry, JHEP 08 (2016) 085 [arXiv: 1606.05239] [INSPIRE]. 
[13] L. Andrianopoli, B.L. Cerchiai, R. D'Auria and M. Trigiante, Unconventional supersymmetry at the boundary of $A d S_{4}$ supergravity, JHEP 04 (2018) 007 [arXiv: 1801.08081] [INSPIRE].

[14] L. Andrianopoli and R. D'Auria, $N=1$ and $N=2$ pure supergravities on a manifold with boundary, JHEP 08 (2014) 012 [arXiv:1405.2010] [INSPIRE].

[15] F. Delduc, C. Lucchesi, O. Piguet and S.P. Sorella, Exact Scale Invariance of the Chern-Simons Theory in the Landau Gauge, Nucl. Phys. B 346 (1990) 313 [InSPIRE].

[16] L.C.Q. Vilar, O.S. Ventura, C.A.G. Sasaki and S.P. Sorella, Algebraic characterization of vector supersymmetry in topological field theories, J. Math. Phys. 39 (1998) 848 [hep-th/9706133] [INSPIRE].

[17] O.M. Del Cima, K. Landsteiner and M. Schweda, Twisted $N=4$ SUSY algebra in topological models of Schwarz type, Phys. Lett. B 439 (1998) 289 [hep-th/9806137] [INSPIRE].

[18] F. Gieres, J. Grimstrup, T. Pisar and M. Schweda, Vector supersymmetry in topological field theories, JHEP 06 (2000) 018 [hep-th/0002167] [INSPIRE].

[19] L. Andrianopoli, B.L. Cerchiai, P.A. Grassi and M. Trigiante, in preparation.

[20] M.M. Caldarelli, R. Emparan and M.J. Rodriguez, Black Rings in (Anti)-deSitter space, JHEP 11 (2008) 011 [arXiv:0806.1954] [INSPIRE].

[21] K. Hosomichi, K.-M. Lee, S. Lee, S. Lee and J. Park, $N=5,6$ Superconformal Chern-Simons Theories and M2-branes on Orbifolds, JHEP 09 (2008) 002 [arXiv:0806.4977] [INSPIRE].

[22] E. Koh, S. Lee and S. Lee, Topological Chern-Simons $\sigma$-model, JHEP 09 (2009) 122 [arXiv: 0907.1641] [INSPIRE].

[23] K. Hosomichi, K.-M. Lee, S. Lee, S. Lee and J. Park, $N=4$ Superconformal Chern-Simons Theories with Hyper and Twisted Hyper Multiplets, JHEP 07 (2008) 091 [arXiv:0805.3662] [INSPIRE].

[24] A. Gallerati, H. Samtleben and M. Trigiante, The $\mathcal{N}>2$ supersymmetric AdS vacua in maximal supergravity, JHEP 12 (2014) 174 [arXiv:1410.0711] [INSPIRE].

[25] G. Inverso, H. Samtleben and M. Trigiante, Type II supergravity origin of dyonic gaugings, Phys. Rev. D 95 (2017) 066020 [arXiv:1612.05123] [INSPIRE].

[26] E. D'Hoker, J. Estes and M. Gutperle, Exact half-BPS Type IIB interface solutions. I. Local solution and supersymmetric Janus, JHEP 06 (2007) 021 [arXiv:0705.0022] [INSPIRE].

[27] E. D'Hoker, J. Estes and M. Gutperle, Exact half-BPS Type IIB interface solutions. II. Flux solutions and multi-Janus, JHEP 06 (2007) 022 [arXiv: 0705.0024] [INSPIRE]. 